\title{
Impact of Layer-by-Layer Self-Assembly Clay-Based Nanocoating on Flame Retardant Properties of Sisal Fiber Cellulose Microcrystals
}

\author{
Chun Wei, ${ }^{1}$ Sihua Zeng, ${ }^{1,2}$ Yuyuan Tan, ${ }^{1}$ Wu Wang, ${ }^{1}$ Jian Lv, ${ }^{1}$ and Hongxia Liu ${ }^{1}$ \\ ${ }^{1}$ Key Laboratory of New Processing Technology for Nonferrous Metals and Materials, Ministry of Education, \\ Ministry-Province Jointly Constructed Cultivation Base for State Key Laboratory of Processing for Nonferrous Metal and \\ Featured Materials, College of Materials Science and Engineering, Guilin University of Technology, Guilin 541004, China \\ ${ }^{2}$ Sichuan EM Technology Co., Ltd., Mianyang 621000, China
}

Correspondence should be addressed to Chun Wei; 1005668130@qq.com

Received 8 August 2014; Revised 30 September 2014; Accepted 14 October 2014

Academic Editor: Hao Wang

Copyright (C) 2015 Chun Wei et al. This is an open access article distributed under the Creative Commons Attribution License, which permits unrestricted use, distribution, and reproduction in any medium, provided the original work is properly cited.

\begin{abstract}
The renewable cationic polyelectrolyte chitosan $(\mathrm{CH})$ and anionic nanomontmorillonite (MMT) layers were alternately deposited on the surface of sisal fiber cellulose microcrystals (SFCM) via layer-by-layer (LBL) self-assembly method. The structure and properties of the composites were characterized by zeta potential, thermal gravimetric analysis (TGA), X-ray diffraction (XRD), field emission scanning electron microscopy (FESEM), Fourier transform infrared spectrometer (FTIR), microcalorimeter (MCC), and so forth. The zeta potential results show that the cellulose microcrystalline surface charge reversed due to the adsorption of $\mathrm{CH}$ and MMT nanoplatelets during multilayer deposition. MMT characteristic diffraction peaks appear in XRD patterns of $\mathrm{SFCM}(\mathrm{CH} / \mathrm{MMT})_{5}$ and SFCM $(\mathrm{CH} / \mathrm{MMT})_{10}$ composites. Additionally, FESEM reveals that the SFCM $(\mathrm{CH} / \mathrm{MMT})_{10}$ surface is covered with a layer of material containing $\mathrm{Si}$, which has been verified by elemental analysis. TGA results show that the initial decomposition (weight loss of 5\%) temperature of SFCM $(\mathrm{CH} / \mathrm{MMT})_{5}$ is increased by $4^{\circ} \mathrm{C}$ compared to that of pure SFCM. On the other hand, carbon residue percentage of SFCM $(\mathrm{CH} / \mathrm{MMT})_{10}$ is $25.1 \%$, higher than that of pure SFCM (5.4\%) by $19.7 \%$. Eventually, it is testified by MCC measurement that CH/MMT coating can significantly reinforce the flame retardant performance of SFCM.
\end{abstract}

\section{Introduction}

Cellulose is the most abundant natural renewable resource $[1,2]$ and has been recently concerned and developed as raw material with the gradual depletion of oil resources and increasing emphasis on environment-friendliness, green materials, and sustainable development. However, these natural fibers have properties of facile thermal degradation and flammability, which limit its application in many fields. Hence, it is necessary to effectively modify cellulose to improve the heat resistance and flame resistance of natural fibers, especially polymer-based reinforced natural fiber composite $[3,4]$.

Layer-by-layer self-assembly (LBL) is a rapidly developed simple and versatile surface modification method in the 90s of the last century $[5,6]$. This facile method can be performed in mild reaction conditions, without limitations of the size and shape of substrate, and it has other advantages: flexible film composition and controllable thickness, stable film performance, and so forth. Thanks to these properties, multilayer films with different characteristics, such as hydrophobicity $[7,8]$, controlled drug release $[9,10]$, chemical sensing $[11,12]$, antibacterial property $[13,14]$, and flame resistance [15-17], can be built through this method.

Montmorillonite (MMT) is typical layered silicate clay consisting of two $\mathrm{Si}-\mathrm{O}$ tetrahedrals and a layer of $\mathrm{Al}-\mathrm{O}$ octahedron amid them. Because partial $\mathrm{Al}^{3+}$ cation within the octahedron are replaced by $\mathrm{Mg}^{2+}$ and others, the surface negative charge among montmorillonite layers is excess, resulting in the fact that $\mathrm{Na}^{+}, \mathrm{Ca}^{2+}$, and other cations are readily absorbed onto montmorillonite layers to maintain the charge balance itself. The unique layered structure and excellent heat 
resistance of MMT make it widely used in the preparation of brand-new high-performance polymer/clay nanocomposites [18]. Compared with conventional filled polymers, MMT can improve mechanical properties [19], gas barrier properties, solvent resistance, and heat resistance of the polymer matrix [20], and a potential flame retardant capacity [21] might be existent.

In this study, a strategy of using sisal fiber cellulose microcrystal (SFCM) as a substrate and alternately adsorbing positively charged polyelectrolyte chitosan $(\mathrm{CH})$ and negatively charged inorganic nanolayer MMT onto the surface of SFCM via LBL technique has been proposed. The effect of $\mathrm{CH} / \mathrm{MMT}$ coating on the thermal performance and flame retardant properties of SFCM was systematically investigated, aiming at applying SFCM to high-performance composite materials.

\section{Experimental}

2.1. Materials. Sisal fibers were obtained from Guangxi Sisal Company, China. Chitosan (medium viscosity of 200$400 \mathrm{mPa} \cdot \mathrm{S}$ ) was purchased from Aladdin Reagent Company. Sodium MMT clay was provided by Zhejiang Fenghong New Materials Co., Ltd., with an average size of $1180 \mathrm{~nm}$. Acetic acid (AR) with a hydrochloric acid (AR) and sulfuric acid (AR) was obtained from Xilong Chemical Co. Both deionized water and sisal fiber microcrystalline cellulose (SFCM) were homemade [22]; SFCM is a rod-like structure with a diameter of about $10 \mu \mathrm{m}$ and a length of about 50-150 um.

2.2. Layer-by-Layer Self-Assembly Method. SFCM charging process is as follows. SFCM was added to an aqueous $\mathrm{H}_{2} \mathrm{SO}_{4}$ solution with a concentration of $45 \%$, and the mixture was then stirred under $50^{\circ} \mathrm{C}$ for $2 \mathrm{~min}$ before it was filtered and washed for 4 times. The precipitation was dried at $50^{\circ} \mathrm{C}$.

MMT solution preparation is as follows. $5 \mathrm{~g}$ of montmorillonite powder was dissolved in $1000 \mathrm{~mL}$ of distilled water by vigorous agitation. The suspension was placed at room temperature for $24 \mathrm{~h}$ of swelling, and then it was processed with ultrasonic sound generated from an ultrasonic cleaner for $3 \mathrm{~h}$. After centrifugation, at a rate of $8000 \mathrm{rad} / \mathrm{min}$ for $5 \mathrm{~min}$, with a high speed centrifuge, the upper clear liquid was collected. SFCMs were alternately adsorbed positively charged polyelectrolyte chitosan $(\mathrm{CH})$ and negatively charged inorganic nanolayer MMT onto the surface of SFCM via LBL technique, as shown in Figure 1.

2.3. Preparation of SFCM $(\mathrm{CH} / \mathrm{MMT})_{n}$ Composites. $0.5 \mathrm{~g}$ of sulfuric acid-treated SFCM was thoroughly dispersed in $100 \mathrm{~mL}$ of $\mathrm{CH}$ solution $(0.2 \mathrm{wt} \%$, containing $1 \mathrm{v} / \mathrm{v} \%$ of acetic acid), which was then magnetically stirred for $10 \mathrm{~min}$. Afterwards, wash and vacuum filtration were alternatively performed for 4 times using $1 \mathrm{v} / \mathrm{v} \%$ of acetic acid solution (dissolved in deionized water). Then, the solid was dispersed into $100 \mathrm{~mL}$ of MMT solution ( $\mathrm{pH}$ not adjusted) and then magnetically stirred for $30 \mathrm{~min}$. After being washed in deionized water for four times, sample was tested for zeta potential measurement. Similarly, SFCM $(\mathrm{CH} / \mathrm{MMT})_{n}$ composite

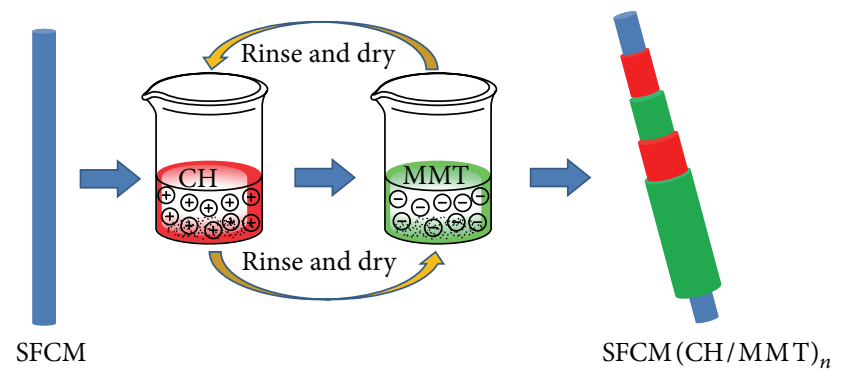

FIGURE 1: Layer-by-layer self-assembly process of $\operatorname{SFCM}(\mathrm{CH} /$ $\mathrm{MMT})_{n}$.

materials ( $n=1,5$, and 10 , resp.) were prepared for the following characterizations.

2.4. Characterization. X-ray diffraction (XRD) spectra were taken on a Holland PANalytical X-Pert PRO X-ray diffractometer with $\mathrm{Cu} \mathrm{K} \alpha$ radiation from $3^{\circ} \sim 40^{\circ}$. Zeta potential was determined using a Zetasizer Nano ZS90 nanoparticle size and zeta potential analyzer (Malvern Company, British). Thermal gravimetric (TGA) test, for the weight loss curve ranging from 50 to $700^{\circ} \mathrm{C}$, was conducted by a NETZSCH STA-449C thermal gravimetric analyzer (NETZSCH Company, Germany) at a heating rate of $10^{\circ} \mathrm{C} / \mathrm{min}$ under the nitrogen. Fourier transformed infrared spectroscopy (FTIR) was obtained using a NICOLETNEXUS 470 FT-IR spectrometer (Perkin-Elmer Company, United States), and samples were prepared using $\mathrm{KBr}$ for flaking. Field emission electron microscopy (FESEM) was operated using a Hitachi S-4800 field emission scanning electron microscope provided by Hitachi High-Technologies Corporation. Microscale combustion calorimetry (MCC) was conducted using an MCC2 (Govmark, Farmingdale, NY) microscale combustion calorimeter, and the sample was heated from 100 to $700^{\circ} \mathrm{C}$ with a heating rate of $1^{\circ} \mathrm{C} / \mathrm{s}$ under the nitrogen atmosphere, and samples are decomposed anaerobically to release volatile combustible gases under high temperatures, which can be combusted in $\mathrm{N}_{2} / \mathrm{O}_{2}(8: 2)$ atmosphere.

\section{Results and Discussion}

3.1. Impact of CH/MMT Coating on SFCM Zeta Potential. Zeta potential online monitoring can be used to characterize the dynamic adsorption of chitosan and montmorillonite suspensions on SFCM surface. The residual CH and MMT solution would affect the zeta potential of the samples, so we can confirm that the measurement of the zeta potential of SFCM/ $(\mathrm{CH} / \mathrm{MMT})_{n}$ adsorption was acted after the samples have been washed in deionized water for some time in order to remove the residual of the $\mathrm{CH}$ or MMT.

Figure 2 shows that the adsorption of each $\mathrm{CH} / \mathrm{MMT}$ layer will turn the negative (or positive) potential into a positive (or negative) one. This potential reverse indicates that $\mathrm{CH} / \mathrm{MMT}$ has been assembled to SFCM surface. Further, as the number of adsorption layers increases, absolute amount of the surface zeta charges becomes gradually smaller. This is 


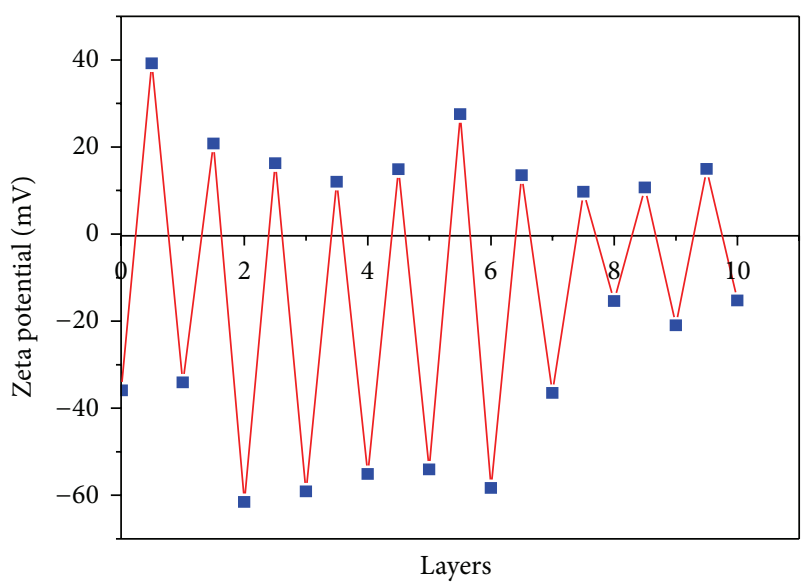

FIGURE 2: Changes of zeta potential at alternative absorption steps of $\mathrm{CH}$ and MMT.

probably because the MMT clay layer deposited on cellulose surface formed a relatively flat deposition surface where there are less nanotopological structures [23] compared with the pure polymer electrolyte self-assembly, so that less polymer chains will be deposited on this plane, resulting in the declined absolute amount of surface zeta charges as above mentioned.

3.2. Effect of CH/MMT Coating on SFCM Composite Crystal Structure. For further describing the assembly of $\mathrm{CH} /$ MMT coating on SFCM surface, XRD was carried out to characterize pure MMT, SFCM, $\mathrm{SFCM}(\mathrm{CH} / \mathrm{MMT})_{5}$, and $\mathrm{SFCM}(\mathrm{CH} / \mathrm{MMT})_{10}$. As shown in Figure 3, between small angles $3^{\circ}$ and $10^{\circ}$, an MMT diffraction peak corresponding to pure MMT at $2 \theta=6.8^{\circ}$ occurs. According to the Bragg equation $(2 d \sin \theta=n \lambda)$, pure MMT interplanar spacing is $13.1 \AA$, whereas an MMT diffraction peak of SFCM $(\mathrm{CH} / \mathrm{MMT})_{5}$ and $\operatorname{SFCM}(\mathrm{CH} / \mathrm{MMT})_{10}$ appears where $2 \theta=4.8^{\circ}$ and $5.0^{\circ}$, which has not been observed in the pattern of pure SFCM. Compared to pure MMT, MMT diffraction peak of $\operatorname{SFCM}(\mathrm{CH} / \mathrm{MMT})_{5}$ and $\mathrm{SFCM}(\mathrm{CH} / \mathrm{MMT})_{10}$ is shifted to a smaller angle. It is elucidated from the peak shift toward small angle that the interplanar spacing of MMT in CH/MMT nanocoating layer is larger than that in pure MMT. The reason might be that $\mathrm{CH}$ amid MMT sheets or the peeling of MMT layer during sonication process increases the distance between layers, which is consistent with $\mathrm{Li}$ et al.s report [24]. Exceptionally, peaks intensity corresponding to $\mathrm{SFCM}(\mathrm{CH} / \mathrm{MMT})_{5}$ and SFCM $(\mathrm{CH} / \mathrm{MMT})_{10}$ is much weaker than the peak intensity of pure MMT. The reason might be the following: the absorbed MMT has a low content and fewer deposition layers, and thus the $\mathrm{CH} / \mathrm{MMT}$ coating is so thin that MMT layered structure, within the coating, cannot be converted from amorphousness into ordered structure [15].

\subsection{Influence of CH/MMT Coating on Thermal Properties} of SFCM. To clarify the impact of CH/MMT nanocoating on thermal performance of SFCM, pure SFCM and $\operatorname{SFCM}(\mathrm{CH} / \mathrm{MMT})_{n}$ composites $(n=1,5$, and 10$)$ were tested
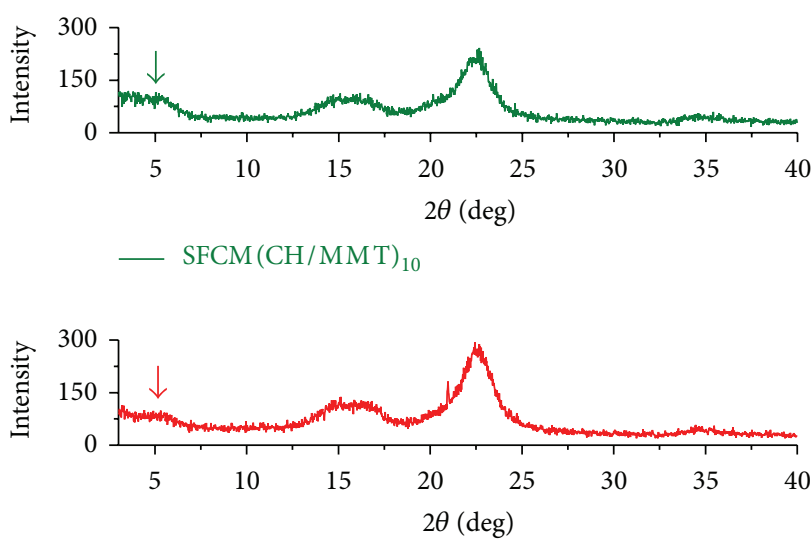

- $\operatorname{SFCM}(\mathrm{CH} / \mathrm{MMT})_{5}$

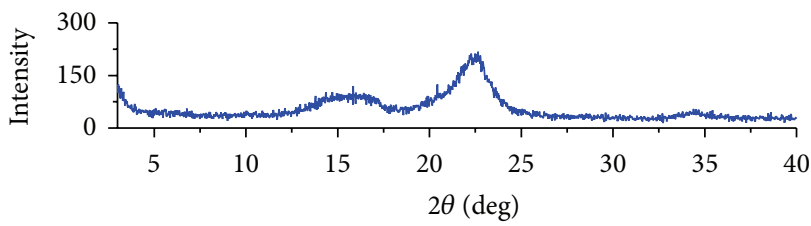

— Pure SFCM

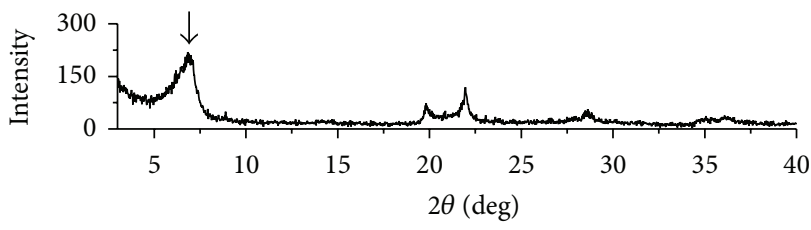

— Pure MMT

FIGURE 3: X-ray diffraction patterns of pure MMT, pure SFCM, $\mathrm{SFCM}(\mathrm{CH} / \mathrm{MMT})_{5}$, and SFCM $(\mathrm{CH} / \mathrm{MMT})_{10}$.

by TGA analysis (Figure 4). Before $340^{\circ} \mathrm{C}$, TGA curves of SFCM, before and after treatment, are almost the same. The characteristic thermal parameters selected were the temperature for $5 \%$ and $10 \%$ weight loss. The results are summarized in Table 1. Table 1 shows that thermal properties of the treated SFCM are slightly better than those of untreated SFCM. At a weight loss of $10 \%$, compared with pure SFCM, decomposition temperatures of SFCM $(\mathrm{CH} / \mathrm{MMT})_{1}, \mathrm{SFCM}(\mathrm{CH} /$ $\mathrm{MMT})_{5}$, and SFCM $(\mathrm{CH} / \mathrm{MMT})_{10}$ composites are elevated by 3,4 , and $3^{\circ} \mathrm{C}$, respectively. On the other hand, between 340 and $700^{\circ} \mathrm{C}$, it is found that the char yield of coated SFCM $(\mathrm{CH} /$ $\mathrm{MMT})_{n}$ is higher than that of pure SFCM. Moreover, as adsorption layers increase, char yield rises as well. At $700^{\circ} \mathrm{C}$, compared with pure SFCM, char yields of SFCM $(\mathrm{CH} / \mathrm{MMT})_{1}$, $\mathrm{SFCM}(\mathrm{CH} / \mathrm{MMT})_{5}$, and $\mathrm{SFCM}(\mathrm{CH} / \mathrm{MMT})_{10}$ are increased by $9.6 \%, 12.0 \%$, and $19.7 \%$, respectively. This increase may be ascribed to the nanolayer of MMT that forms a sheet of carbon residue covering the surface of cellulose during heating process, because of which heat was insulated, protecting an amount of cellulose microcrystals from destruction.

3.4. Fourier Transform Infrared (FTIR) Analysis. Carbon residue, after TGA test, of pure SFCM, SFCM $(\mathrm{CH} / \mathrm{MMT})_{5}$, and $\operatorname{SFCM}(\mathrm{CH} / \mathrm{MMT})_{10}$ was subjected to FTIR testing. As it is shown in Figure 5, for TGA residual carbon of 


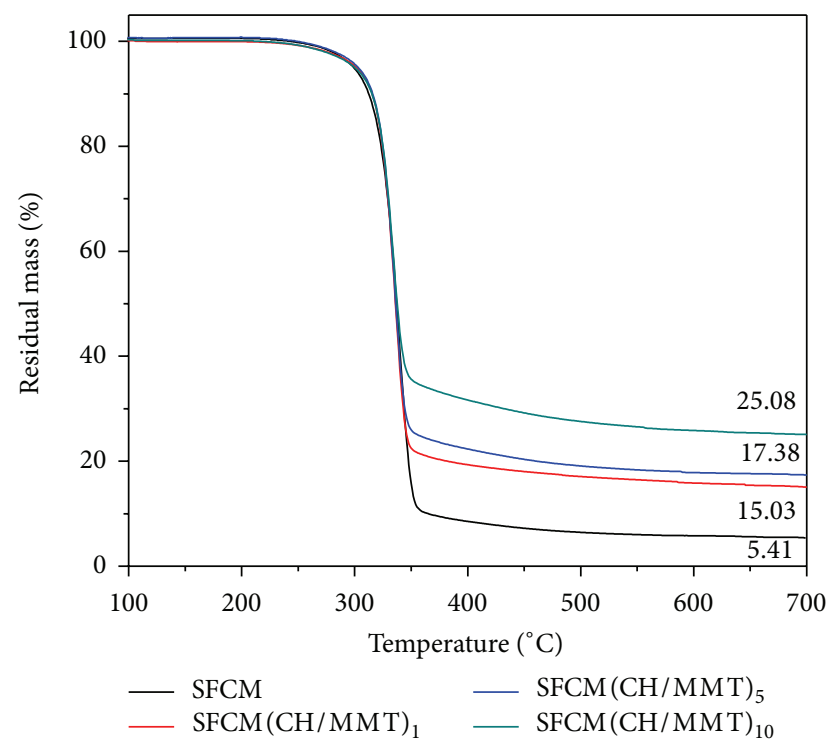

FIGURE 4: TGA plots of SFCM coated with different number of $\mathrm{CH} / \mathrm{MMT}$ bilayers.

TABLE 1: TGA results for SFCM coated with different number of $\mathrm{CH} / \mathrm{MMT}$ bilayers.

\begin{tabular}{lccc}
\hline Samples & $\begin{array}{c}\text { Temperature at } \\
5 \mathrm{wt} \% \text { loss }\left({ }^{\circ} \mathrm{C}\right)\end{array}$ & $\begin{array}{c}\text { Temperature at } \\
\text { 10 wt\% loss }\left({ }^{\circ} \mathrm{C}\right)\end{array}$ & $\begin{array}{c}\text { Residue } \\
(\mathrm{wt} \%)\end{array}$ \\
\hline SFCM & 299 & 312 & 5.4 \\
$\mathrm{SFCM}(\mathrm{CH} / \mathrm{MMT})_{1}$ & 302 & 315 & 15.0 \\
$\mathrm{SFCM}(\mathrm{CH} / \mathrm{MMT})_{5}$ & 303 & 316 & 17.4 \\
$\mathrm{SFCM}(\mathrm{CH} / \mathrm{MMT})_{10}$ & 300 & 315 & 25.1 \\
\hline
\end{tabular}

$\mathrm{SFCM}(\mathrm{CH} / \mathrm{MMT})_{10}$, stretching vibration peaks corresponding to methyl and methylene on cellulose appeared at 2970 and $2925 \mathrm{~cm}^{-1}$ [25], while these peaks of pure SFCM and $\mathrm{SFCM}(\mathrm{CH} / \mathrm{MMT})_{5}$ are almost negligible. This implies that, with the increase of $\mathrm{CH} / \mathrm{MMT}$ layers, cellulose microcrystals was protected in the process of thermal degradation. In addition, there is another peak at $1050 \mathrm{~cm}^{-1}$ for TG residual carbon of $\operatorname{SFCM}(\mathrm{CH} / \mathrm{MMT})_{5}$ and $\operatorname{SFCM}(\mathrm{CH} / \mathrm{MMT})_{10}$, which may be $\mathrm{Si}-\mathrm{O}-\mathrm{Si}$ stretching vibration peak of MMT or stretching vibration absorption peak of the cellulose C-C skeleton, indicating that the TG residual carbon of $\mathrm{SFCM}(\mathrm{CH} / \mathrm{MMT})_{10}$ might be composed of cellulose microcrystalline and MMT [26].

3.5. Morphology and Surface Element Analysis of SFCM and $\operatorname{SFCM}(\mathrm{CH} / \mathrm{MMT})_{10}$ Composite. Field emission scanning electron micrographs of pure SFCM (Figure 6(a)) and its TGA carbon residue (Figure 6(c)) and SFCM(CH/MMT) 10 (Figure 6(b)) and its TGA carbon residue (Figure 6(d)) are given. It can be observed that the surface of pure SFCM is continuous cellulose structure with clear texture, clean surface, and regular structure, whereas the cellulose surface is coated with fold-like layers in Figure 6(b) and it is unable to clearly see continuous and clear-textured cellulose skeleton. For this reason, surface element analysis for pure SFCM and

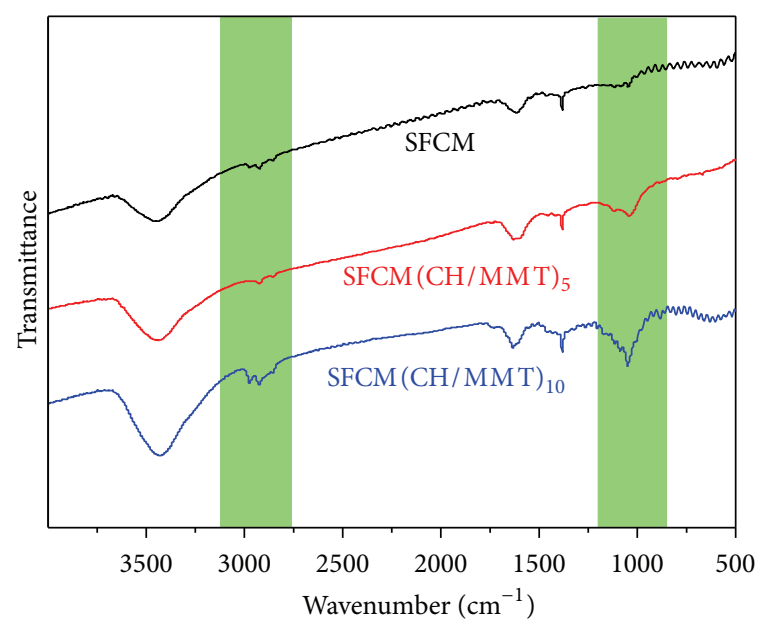

FIGURE 5: FTIR spectra of char residues of pure SFCM, $\mathrm{SFCM}(\mathrm{CH} / \mathrm{MMT})_{5}$, and $\operatorname{SFCM}(\mathrm{CH} / \mathrm{MMT})_{10}$ (charred in $\mathrm{N}_{2}$ at $\left.700^{\circ} \mathrm{C}\right)$.

SFCM $(\mathrm{CH} / \mathrm{MMT})_{10}$ samples was shown in Figures $7(\mathrm{a})$ and 7(b). Figure 7(a) verifies the presence of elements $C$ and $\mathrm{O}$ on the surface of pure SFCM without Si. However, in Figure 7(b), besides $\mathrm{C}$ and $\mathrm{O}, \mathrm{Si}, \mathrm{Mg}, \mathrm{Al}$, and other elements were also detected on the surface of $\operatorname{SFCM}(\mathrm{CH} / \mathrm{MMT})_{10}$, demonstrating that the fold layers in Figure 6(b) should be MMT nanolayers.

Clear spiral contraction texture of pure SFCM can be observed in Figure 6(c), and this is because of the severe contraction of cellulose during heating process. On the other hand, composite SFCM $(\mathrm{CH} / \mathrm{MMT})_{10}$ surface shrinkage has been significantly weakened, and this may be due to a thermal insulation coke layer which was formed during the heating of CH/MMT coating. This layer has satisfying insulation properties, inhibiting the heat from being transferred to the internal cellulose, that is, a protective effect.

3.6. Impact of CH/MMT Coating on Flame Retardant Performance of SFCM. To study the effect of CH/MMT on combustion properties of pure SFCM, SFCM $(\mathrm{CH} / \mathrm{MMT})_{5}$, and $\mathrm{SFCM}(\mathrm{CH} / \mathrm{MMT})_{10}$, powder samples were tested through microscale combustion calorimetry (MCC). The combustion properties of the samples will be characterized on the basis of the released heat, heat release rate, and other parameters. In Figure 8 and Table 2, compared with pure SFCM, both temperature of initial decomposition and heat release rate of SFCM $(\mathrm{CH} / \mathrm{MMT})_{5}$ and $\mathrm{SFCM}(\mathrm{CH} / \mathrm{MMT})_{10}$ were slightly decreased. Moreover, both total released heat and maximum heat release rate of $\operatorname{SFCM}(\mathrm{CH} / \mathrm{MMT})_{5}$ and $\mathrm{SFCM}(\mathrm{CH} / \mathrm{MMT})_{10}$ composites are lower than those of pure SFCM, where the maximum heat release rate of $\mathrm{SFCM}(\mathrm{CH} / \mathrm{MMT})_{10}$ is lower than that of pure cellulose by $47.5 \mathrm{~W} / \mathrm{g}$, and the total released heat of $\mathrm{SFCM}(\mathrm{CH} / \mathrm{MMT})_{5}$ and SFCM $(\mathrm{CH} / \mathrm{MMT})_{10}$ is lower than that of pure SFCM by more than $21 \%$.

It is concluded that the presence of $\mathrm{CH} / \mathrm{MMT}$ coating enhanced the flame retardant performance of SFCM to some 


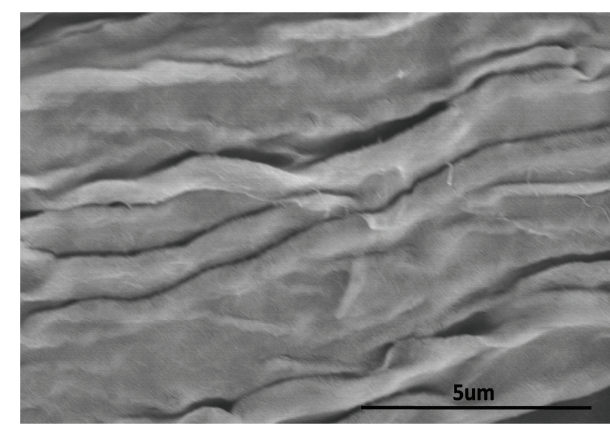

(a)

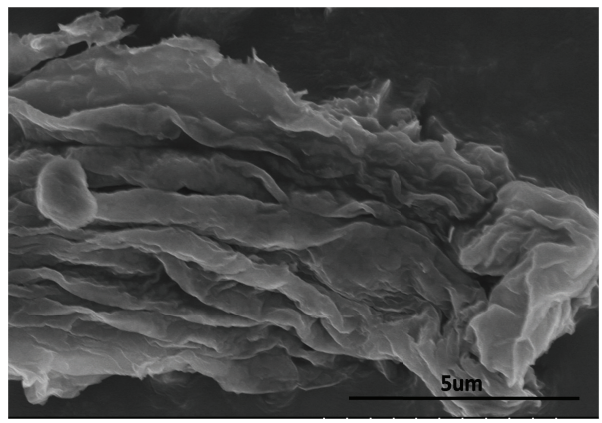

(c)

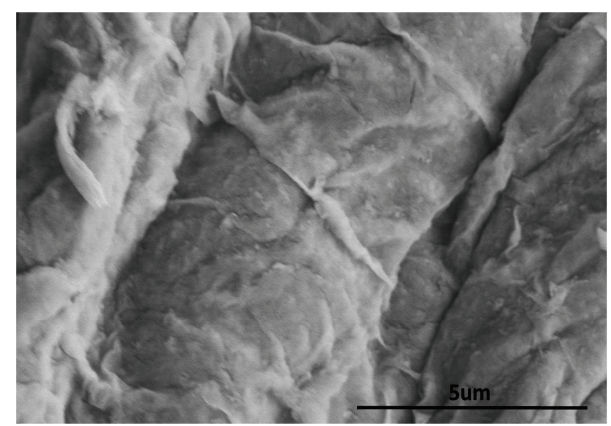

(b)

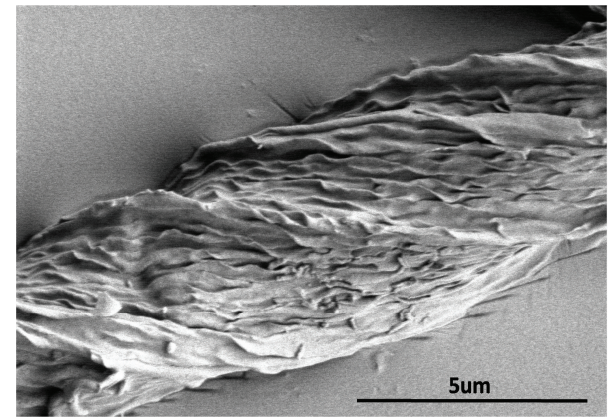

(d)

Figure 6: FESEM images of (a) pure SFCM, (b) SFCM(CH/MMT) $)_{10}$, and (c) char residues of pure SFCM and (d) SFCM(CH/MMT) 10 (charred in $\mathrm{N}_{2}$ at $700^{\circ} \mathrm{C}$ ).

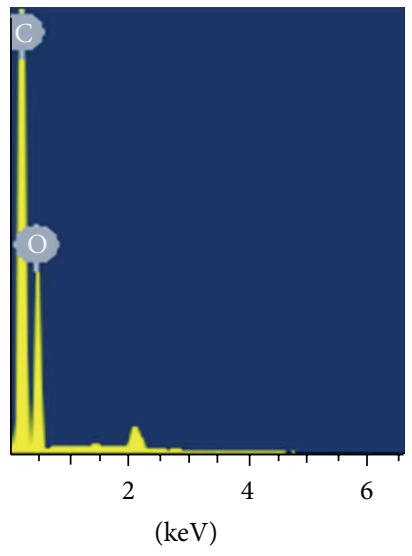

(a)

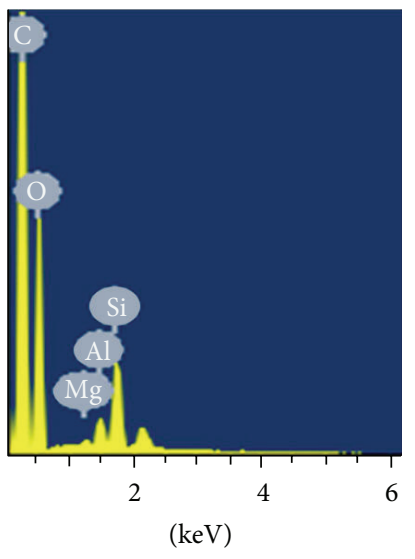

(b)
Figure 7: Superficial elemental distribution of (a) pure SFCM and (b) $\operatorname{SFCM}(\mathrm{CH} / \mathrm{MMT})_{10}$.

extent. It is elucidated that MMT inorganic layer does not react with the cellulose, so MMT mainly functions as a physical barrier on the surface of cellulose, during the combustion process of $\operatorname{SFCM}(\mathrm{CH} / \mathrm{MMT})_{n}$, of which flame retardant effect is limited. Hence, in terms of flame retardant performance, the composites cannot match with intumescent $\mathrm{P}-\mathrm{N}$ fire retardant that can react with cellulose [27], which has also been confirmed by literatures related to retardant coating fabricated by inorganic nanoparticles, such as $\mathrm{SiO}_{2}$ [28] or POSS [29].

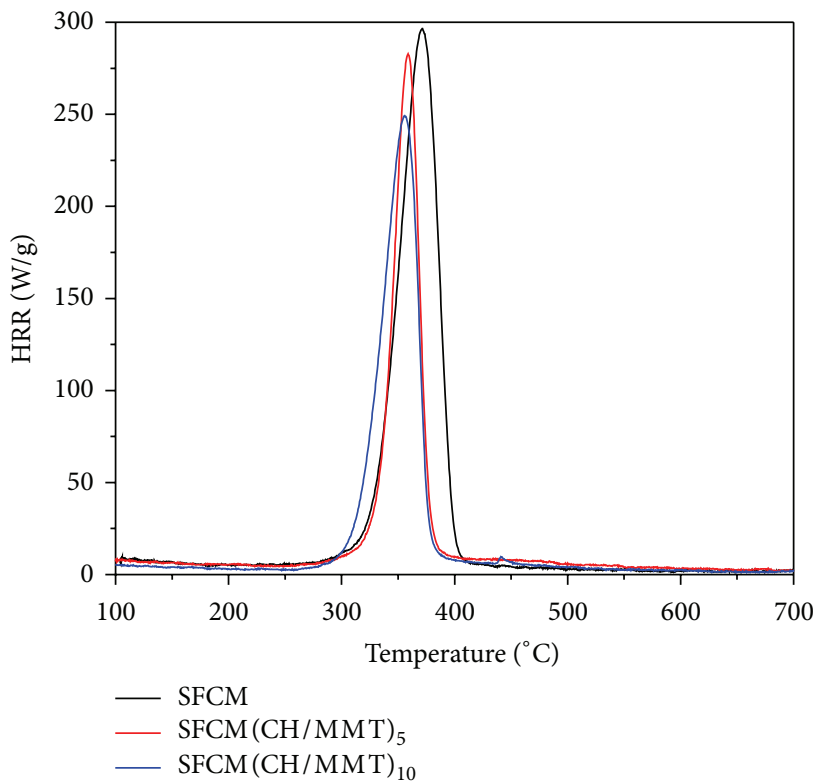

FIGURE 8: Heat release rate versus temperature for pure SFCM, $\mathrm{SFCM}(\mathrm{CH} / \mathrm{MMT})_{5}$, and SFCM $(\mathrm{CH} / \mathrm{MMT})_{10}$.

\section{Conclusion}

$\mathrm{CH} / \mathrm{MMT}$ coating on the surface of SFCM was prepared by LBL self-assembly method and our results reveal that the assembly of $\mathrm{CH} / \mathrm{MMT}$ coating could increase heat resistance 
TABLE 2: MCC results of pure SFCM, $\operatorname{SFCM}(\mathrm{CH} / \mathrm{MMT})_{5}$, and $\mathrm{SFCM}(\mathrm{CH} / \mathrm{MMT})_{10}$.

\begin{tabular}{lccc}
\hline Samples & $\begin{array}{c}\text { pkHRR } \\
\left({ }^{\circ} \mathrm{C}\right)\end{array}$ & $\begin{array}{c}\text { pkHRR } \\
(\mathrm{W} / \mathrm{g})\end{array}$ & $\begin{array}{c}\text { Total HR } \\
(\mathrm{kJ} / \mathrm{g})\end{array}$ \\
\hline SFCM & 371.2 & 296.6 & 15.0 \\
SFCM $(\mathrm{CH} / \mathrm{MMT})_{5}$ & 318.2 & 282.7 & 11.3 \\
SFCM $(\mathrm{CH} / \mathrm{MMT})_{10}$ & 302.4 & 249.1 & 11.7 \\
\hline
\end{tabular}

and flame retardancy of SFCM. TGA results demonstrate that the thermal performance of treated SFCM is commonly superior to that of untreated SFCM. With the increase of the number of adsorbed layers, carbon residue increases. Meanwhile, MCC results indicate that the existence of $\mathrm{CH} / \mathrm{MMT}$ coating has significantly improved flame retardant capability of SFCM.

\section{Conflict of Interests}

The authors declare that there is no conflict of interests regarding the publication of this paper.

\section{Acknowledgments}

The authors would like to acknowledge the National Natural Science Foundation of China (Grant nos. 21264005, 21204013, 51263005, and 51163003), Guangxi Natural Science Foundation of China (Grant nos. 2013GXNSFDA019008 and 2014GXNSFAA118321), Guangxi Small Highland Innovation Team of Talents in Colleges and Universities, and Innovation Team of Guangxi Universities' Talent Highland for financial support.

\section{References}

[1] X. F. Li, E. Y. Ding, and G. K. Li, "A method of preparing spherical nano-crystal cellulose with mixed crystalline forms of cellulose I and II," Chinese Journal of Polymer Science, vol. 19, no. 3, pp. 291-296, 2001.

[2] M. Larssona, Q. Zhou, and A. Larssona, "Different types of microfibrillated cellulose as filler materials in polysodium acrylate superabsorbents," Chinese Journal of Polymer Science, vol. 29, no. 4, pp. 407-413, 2011.

[3] A. Sutka, S. Kukle, J. Gravitis, R. Milašius, and J. Malašauskiene, "Nanofibre electrospinning poly(vinyl alcohol) and cellulose composite mats obtained by use of a cylindrical electrode," Advances in Materials Science and Engineering, vol. 2013, Article ID 932636, 6 pages, 2013.

[4] B. Madsen and E. K. Gamstedt, "Wood versus plant fibers: similarities and differences in composite applications," Advances in Materials Science and Engineering, vol. 2013, Article ID 564346, 14 pages, 2013.

[5] Y. Wang, A. S. Angelatos, and F. Caruso, "Template synthesis of nanostructured materials via layer-by-layer assembly," Chemistry of Materials, vol. 20, no. 3, pp. 848-858, 2008.

[6] X. Zhang, H. Chen, and H. Y. Zhang, "Layer-by-layer assembly: from conventional to unconventional methods," Chemical Communications, no. 14, pp. 1395-1405, 2007.
[7] J. Bravo, L. Zhai, Z. Wu, R. E. Cohen, and M. F. Rubner, "Transparent superhydrophobic films based on silica nanoparticles," Langmuir, vol. 23, no. 13, pp. 7293-7298, 2007.

[8] R. M. Jisr, H. H. Rmaile, and J. B. Schlenoff, "Hydrophobic and ultrahydrophobic multilayer thin films from perfluorinated polyelectrolytes," Angewandte Chemie, vol. 44, no. 5, pp. 782785, 2005.

[9] K. C. Wood, J. Q. Boedicker, D. M. Lynn, and P. T. Hammond, "Tunable drug release from hydrolytically degradable layer-bylayer thin films," Langmuir, vol. 21, no. 4, pp. 1603-1609, 2005.

[10] Y. Yan, G. K. Such, A. P. R. Johnston, H. Lomas, and F. Caruso, "Toward therapeutic delivery with layer-by-layer engineered particles," ACS Nano, vol. 5, no. 6, pp. 4252-4257, 2011.

[11] Q. Gao, Y. Y. Guo, W. Y. Zhang, H. L. Qi, and C. X. Zhang, "An amperometric glucose biosensor based on layer-by-layer GOxSWCNT conjugate/redox polymer multilayer on a screenprinted carbon electrode," Sensors and Actuators B: Chemical, vol. 153, no. 1, pp. 219-225, 2011.

[12] A. Salimi and A. Noorbakhsh, "Layer by layer assembly of glucose oxidase and thiourea onto glassy carbon electrode: fabrication of glucose biosensor," Electrochimica Acta, vol. 56, no. 17, pp. 6097-6105, 2011.

[13] C. M. Dvoracek, G. Sukhonosova, M. J. Benedikt, and J. C. Grunlan, "Antimicrobial behavior of polyelectrolyte-surfactant thin film assemblies," Langmuir, vol. 25, no. 17, pp. 10322-10328, 2009.

[14] J. A. Lichter, K. J. van Vlietpa, and M. F. Rubner, "Design of antibacterial surfaces and interfaces: polyelectrolyte multilayers as a multifunctional platform," Macromolecules, vol. 42, no. 22, pp. 8573-8586, 2009.

[15] G. Laufer, F. Carosio, R. Martinez, G. Camino, and J. C. Grunlan, "Growth and fire resistance of colloidal silica-polyelectrolyte thin film assemblies," Journal of Colloid and Interface Science, vol. 356, no. 1, pp. 69-77, 2011.

[16] Y.-C. Li, S. Mannen, A. B. Morgan et al., "Intumescent all-polymer multilayer nanocoating capable of extinguishing flame on fabric," Advanced Materials, vol. 23, no. 34, pp. 3926-3931, 2011.

[17] A. Laachachi, V. Ball, K. Apaydin, V. Toniazzo, and D. Ruch, "Diffusion of polyphosphates into (poly(allylamine)-montmorillonite) multilayer films: flame retardant-intumescent films with improved oxygen barrier," Langmuir, vol. 27, no. 22, pp. 13879-13887, 2011.

[18] M. Durandish and A. Alipour, "Investigation into morphology, microstructure and properties of SBR/EPDM/ORGANO montmorillonite nanocomposites," Chinese Journal of Polymer Science (English Edition), vol. 31, no. 4, pp. 660-669, 2013.

[19] H. Yang and Q. Zheng, "Structure stability of organic montmorillonite used for preparing polyethylene/montmorillonite nanocomposite," Journal of Materials Science Letters, vol. 22, no. 20, pp. 1431-1433, 2003.

[20] L. Xia, J. Xiong, B. Shentu, and Z. Weng, “Thermal-oxidative degradation and accelerated aging behavior of polyamide 6/epoxy resin-modified montmorillonite nanocomposites," Journal of Applied Polymer Science, vol. 131, no. 19, 2014.

[21] X. J. Zhang, F. Ren, L. Q. Zhang, D. Dong, K. Jiang, and Y. Lu, "Effects of the loading levels of organically modified montmorillonite on the flame-retardant properties of asphalt," Journal of Applied Polymer Science, vol. 131, no. 21, 2014.

[22] J. I. Morán, V. A. Alvarez, V. P. Cyras, and A. Vázquez, "Extraction of cellulose and preparation of nanocellulose from sisal fibers," Cellulose, vol. 15, no. 1, pp. 149-159, 2008. 
[23] G. Laufer, M. A. Priolo, C. Kirkland, and J. C. Grunlan, "High oxygen barrier, clay and chitosan-based multilayer thin films: an environmentally-friendly foil replacement," Green Materials, vol. 1, no. 1, pp. 4-10, 2013.

[24] Y.-C. Li, J. Schulz, S. Mannen et al., "Flame retardant behavior of polyelectrolyte-clay thin film assemblies on cotton fabric," ACS Nano, vol. 4, no. 6, pp. 3325-3337, 2010.

[25] Z. Lin, S. Renneckar, and D. P. Hindman, "Nanocompositebased lignocellulosic fibers 1 . Thermal stability of modified fibers with clay-polyelectrolyte multilayers," Cellulose, vol. 15, no. 2, pp. 333-346, 2008.

[26] T. Zhang, H. Yan, M. Peng, L. Wang, H. Ding, and Z. Fang, "Construction of flame retardant nanocoating on ramie fabric via layer-by-layer assembly of carbon nanotube and ammonium polyphosphate," Nanoscale, vol. 5, no. 7, pp. 3013-3021, 2013.

[27] G. Laufer, C. Kirkland, A. B. Morgan, and J. C. Grunlan, "Intumescent multilayer nanocoating, made with renewable polyelectrolytes, for flame-retardant cotton," Biomacromolecules, vol. 13, no. 9, pp. 2843-2848, 2012.

[28] F. Carosio, G. Laufer, J. Alongi, G. Camino, and J. C. Grunlan, "Layer-by-layer assembly of silica-based flame retardant thin film on PET fabric," Polymer Degradation and Stability, vol. 96, no. 5, pp. 745-750, 2011.

[29] Y.-C. Li, S. Mannen, J. Schulz, and J. C. Grunlan, "Growth and fire protection behavior of POSS-based multilayer thin films," Journal of Materials Chemistry, vol. 21, no. 9, pp. 3060-3069, 2011. 

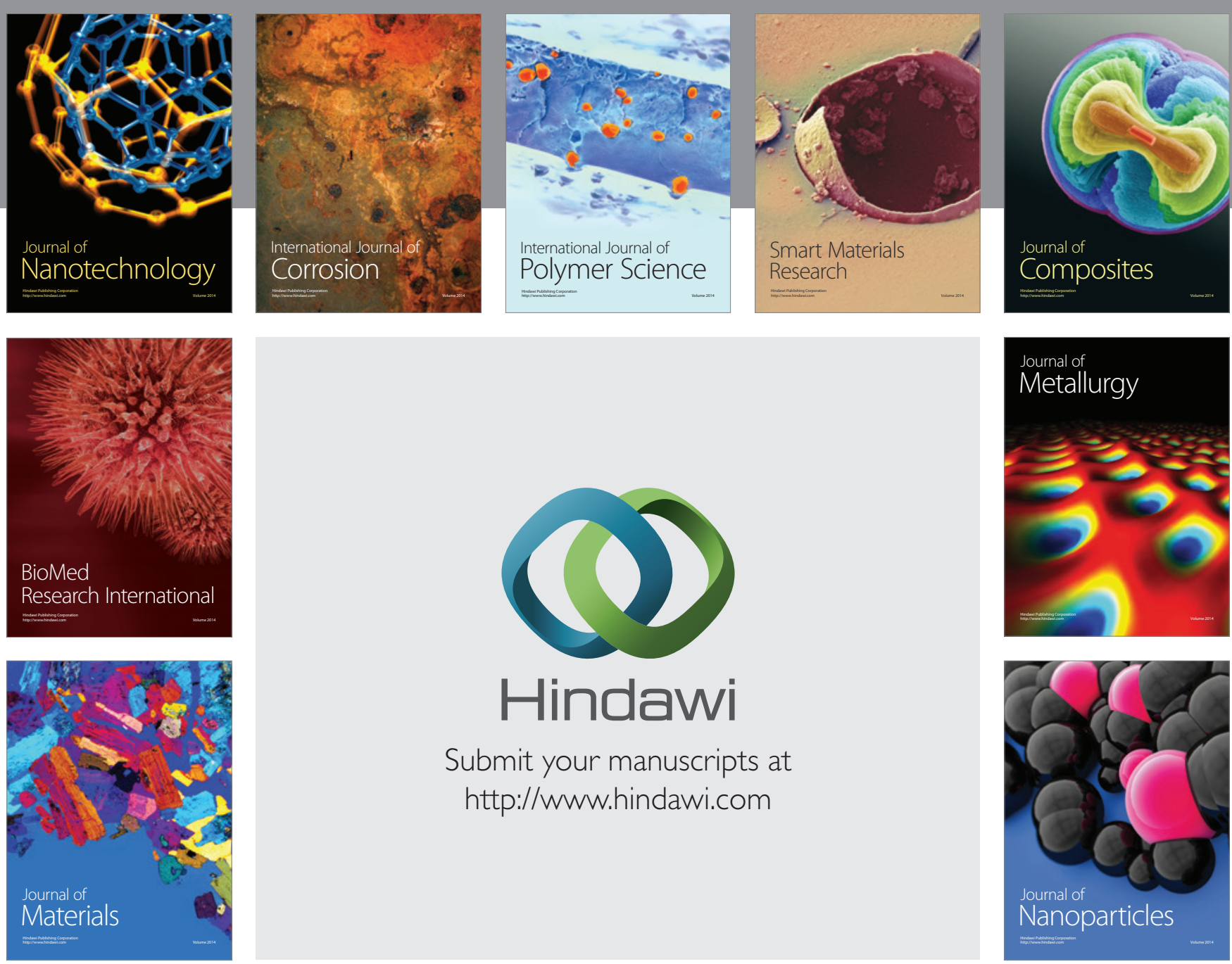

Submit your manuscripts at http://www.hindawi.com
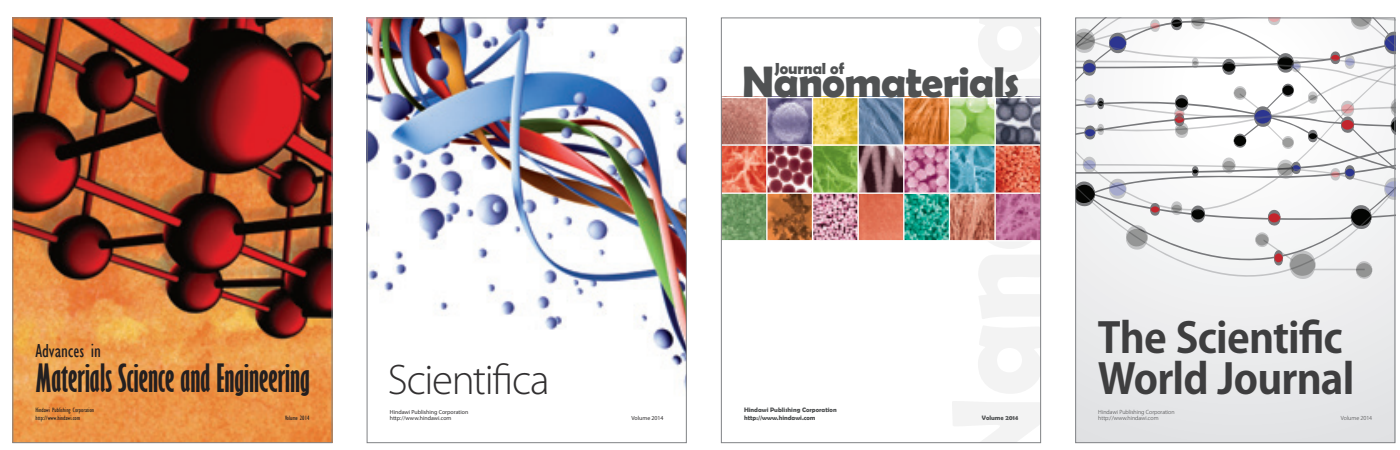

\section{The Scientific World Journal}
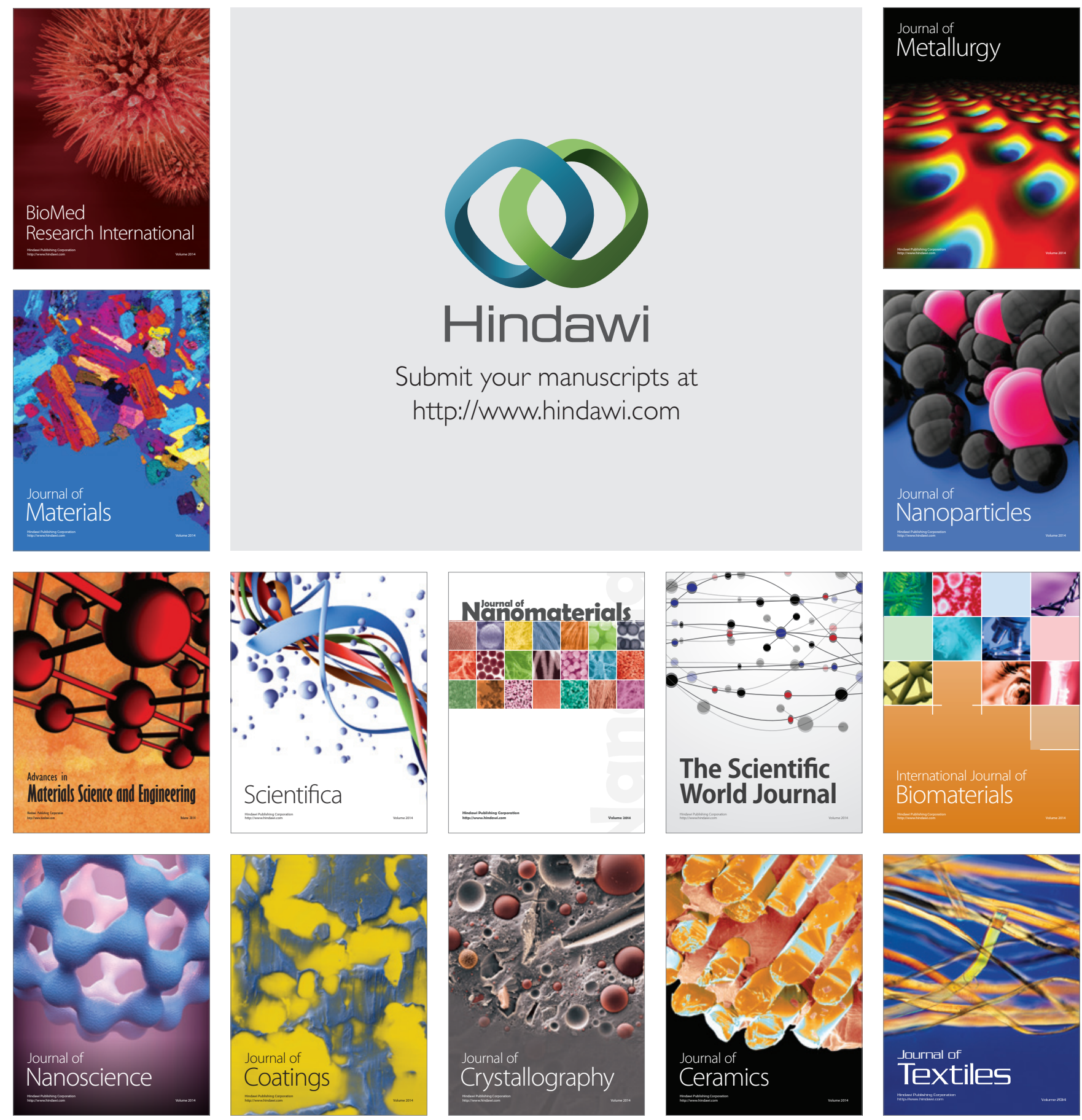